\title{
Uterine Corpus Endometrial Stromal and Related Neoplasms
}

National Cancer Institute

\section{Source}

National Cancer Institute. Uterine Corpus Endometrial Stromal and Related Neoplasms.

NCl Thesaurus. Code C40217.

A category of benign or malignant mesenchymal neoplasms that arises from the uterine corpus. This category includes the endometrial stromal nodule which is benign and noninvasive, the endometrial stromal sarcoma, and the undifferentiated uterine sarcoma. The endometrial stromal sarcoma is an invasive malignant neoplasm, further subdivided into low grade and high grade endometrial stromal sarcoma. Undifferentiated uterine sarcoma was previously also known as high grade endometrial stromal sarcoma. In 2014, high grade endometrial stromal sarcoma was reclassified and is currently considered a distinct and rare neoplasm. It appears to have a prognosis that falls between low grade endometrial stromal sarcoma and undifferentiated sarcoma. 